\title{
Erratum
}

\section{Insulin resistance is a risk factor for progression to Type 1 diabetes}

\author{
S. Fourlanos ${ }^{1}$ P. Narendran ${ }^{1,2}$ • G. B. Byrnes ${ }^{3}$ P. G. Colman ${ }^{4}$ L. C. Harrison ${ }^{1}$ \\ ${ }^{1}$ Autoimmunity and Transplantation Division, The Walter and Eliza Hall Institute of Medical Research, Parkville, Victoria, \\ Australia \\ 2 Department of Clinical Science, University of Bristol, UK \\ ${ }^{3}$ Centre for Genetic Epidemiology, University of Melbourne, Victoria, Australia \\ ${ }^{4}$ Department of Diabetes and Endocrinology, Royal Melbourne Hospital, Parkville, Victoria, Australia
}

\section{Diabetologia (2004) 47:1661- 1667}

Owing to a mistake by the authors, the y-axis on Fig. 3 was incorrectly labelled 'Proportion developing diabetes'. It should have read: 'Proportion diabetesfree', as below.

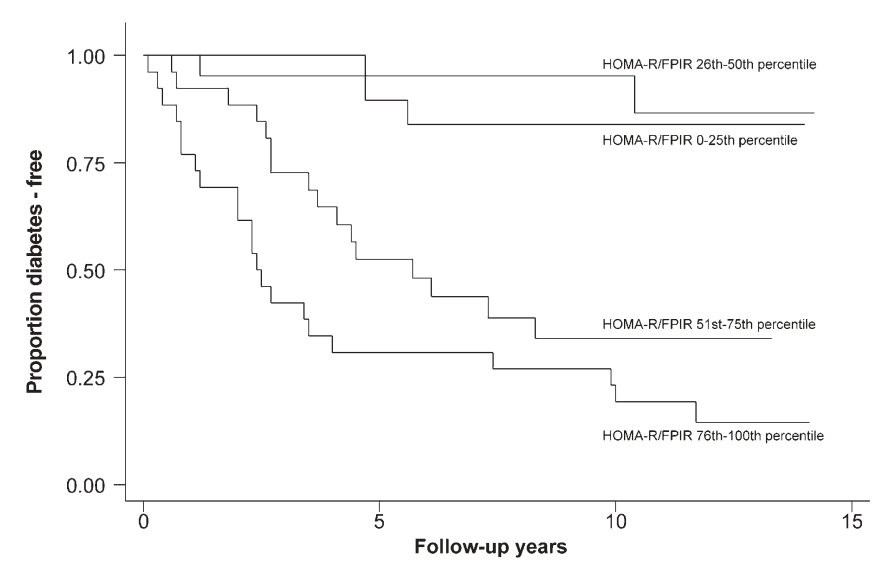

Fig. 3. Kaplan-Meier survival estimates according to baseline HOMA-R : FPIR quartile

Published online: 11 December 2004

(C) Springer-Verlag 2004

DOI 10.1007/s00125-004-1621-2

L. C. Harrison $(\bowtie)$

Autoimmunity and Transplantation Division,

The Walter and Eliza Hall Institute of Medical Research,

$1 G$ Royal Parade, Parkville, Victoria 3050, Australia

E-mail: harrison@wehi.edu.au

Tel.: +61-3-93452460, Fax: +61-3-93470852

The online version of the original article can be found at http://dx.doi.org/10.1007/s00125-004-1507-3 